\title{
Sites of Anesthetic Block of the Lateral and Medial Palmar Metacarpal Nerves in Equines of Indeterminate Breed
}

\author{
Tiago Machado Vieira', Henrique Inhauser Riceti Magalhães ${ }^{2,3}$, Ygor Henrique de Paula ${ }^{3}$, \\ Fabiano Braz Romão ${ }^{3}$, Jeferson Borges Barcelos ${ }^{3,4}$, Gabriela Soares de Moura Guenka ${ }^{5}$, \\ Lucas de Assis Ribeiro ${ }^{3}$ \& Frederico Ozanam Carneiro e Silva ${ }^{1,3}$
}

\begin{abstract}
Background: Local anesthesia blockage at equines claudication diagnosis besides favoring the beginning of the therapy and relieving patient's pain, it allows the identification of the specific affected region and its origin, however, if it is done incorrectly (by improper volume of anesthetics application and/or mistakenly nerve identification due to the lack of anatomic referential) it may cause inflammation, infection or even tissue necrosis. The aim was describing anatomically lateral and medial palmar metacarpal nerves in equines to improve diagnostic anesthetic block techniques on four metacarpophalangeal articulation joints of the specie.

Materials, Methods \& Results: A number of 20 thoracic limbs from 10 equines of indeterminate breed were used to be fixated in $10 \%$ phormaldehyde and with a marked arterial system by water solution of red dyed latex. The structures were identified by dissection, and their denomination bases on Nomina Anatomica Veterinaria (N.A.V.) by the International Committee on Veterinary Gross Anatomical Nomenclature. The study had been approved by the Board of Ethics in Animal Use of the University Center of Patos de Minas (UNIPAM), protocol n ${ }^{\circ} 18 / 17$. The lateral and medial palmar metacarpal nerves have been observed in all of the specimens originated from the deep branch of lateral palmar nerve, immediately distal to the intercarpal articulation. After its origin, the first nerve stretched ipsilaterally from the common trunk, whilst the second nerve positioned counter-lateral manner after superficially crossing the interosseus muscle. Both penetrated at the level of the mid third of the referred muscle and the metacarpal bone III and, assumed respectively a parallel medial position to the metacarpal bone IV and lateral to the metacarpal II, being intimately associated to the palmar face of the metacarpal bone III. Distally, those nerves emerged from a palmar position to a lateral and medial to the distal extremity of the metacarpal bones, corresponding and adjacent to the metacarpophalangeal articulation palmar recession, where they had finally ramified to partially innervate the associated articular capsule and the subcutaneous tissue of the region. Discussion: The lateral and medial palmar metacarpal nerves present similar origin and disposition to the above described, however, besides largely mentioned in literature, we point out here as recommended by N.A.V., no use of terms like axial and abaxial as position indicators for equine. As a part of block anesthetic diagnosis of four metacarpophalangeal articulation points, it is hereby suggested for the studied nerves desensitization, should be palpated the distal extremities of the metacarpal bones IV and II. After the identification, using thumb located from the extremity at proximal direction way, introduce the needle bilaterally for $1.5 \mathrm{~cm}$ downright between the above mentioned metacarpal bones and the interosseus muscle, being these latter and the flexor muscle tendons drawn in the palmar direction. Thus, it becomes valid to affirm that the technique of four-point diagnostic anesthetic block of the metacarpophalangeal articulation has been perfected for equines, and that it will limit the chances of false results on account of misplaced puncturing of nearby structures, such as, for example, the synovial recesses, mentioned as the location of the utmost gravity, hence septic synovitis may come in play.
\end{abstract}

Keywords: claudication, Equidae, metacarpophalangeal articulation, palmar metacarpal nerves. 


\section{INTRODUCTION}

Equines are mammals which have been fascinating man for centuries and whose evolutionary history mingles with that of the development of civilization. Today, are resorted to in a myriad of forms: transportation, public protection, sports and in medicine - to name but a few [5].

The performance of equines is always benefits from the integrity of their locomotor system, which delivers a high capacity of support [3]. Yet, locomotor problems are the main complaint in veterinary consultations within equine breeding [6] and, within that context, upon the presence of musculoskeletal pain, claudication comes along [18].

According to Baxter [2], the majority of the cases of limping takes place in the thoracic limbs, and nearly $95 \%$ of those pictures are related to the distal portions of the carpus [12]. Besides the complete anamnesis, local anesthesia is one of the most widely used tools in equine clinical practice in order to have claudication diagnosed [14,18] and is performed to locate the origin of the pain causing the symptom [8].

A number of factors may interfere with the efficiency of the procedure - from the inaccurate location of the nerve and an improper volume of anesthetic [9], to intravascular and intra-articular administration [16] and the lack of anatomic references.

Thus, it has been sought to evaluate the morphology of the lateral and medial palmar metacarpal nerves in equines of indeterminate breed with aims at suggest a technique that is proximal to the ones already described and perfecting the four-point diagnostic anesthetic block of the metacarpophalangeal articulation of the species.

\section{MATERIALS AND METHODS}

Twenty thoracic limbs have been used out of 10 adult equines of indeterminate breed specimens of Equus caballus with no distinction of gender. The material used originates from the Laboratory of Animal Anatomy of the University Center of Patos de Minas (UNIPAM) (Patos de Minas, MG, Brazil).

As a routine methodology, all of the samples have had their arterial systems marked for identification after distal cannulation of the median artery. To that end, the same had been filled with latex aqueous solution $^{1}$ at $50 \%$, stained with specific red pigment ${ }^{2}$. Now, the fixation took place by means of intramuscular and subcutaneous injections of an aqueous solution of formaldehyde at $10 \%{ }^{3}$, so they would subsequently be immersed in opaque tanks containing the same solution.

Dissection commenced by means of a longitudinal incision of the skin, from the medial face of the proximal row of the carpal bones to the medial face of the proximal interphalangeal articulation. The skin and the subcutaneous screen which enfolded the entire distal extremity of the limb were lifted as from the initial incision. The ulnar and median nerves were respectively identified and dissected in a first moment, at the caudal and medial face of the forearm.

The dorsal branch of the ulnar nerve was isolated until the contour, in the caudal-dorsal direction, of the tendon of the lateral ulnar muscle. Yet, the palmar branch of the ulnar nerve was dissected until its communication, at the level of the radioulnar-carpal articulation, with the lateral palmar nerve - a branch derived from the median nerve. As mentioned above, from the dissection of the median nerve on the medial face of the carpus, in varying regions, it has been possible to identify the subdivision of the same in lateral and medial palmar nerves.

After the identification of the lateral palmar nerve - immediately distal to the intercarpal articulation - the origin of the deep branch of the lateral palmar nerve, which extended from the lateral palmar nerve towards the palmar face of the metacarpal bone III, had been visualized. The deep branch of the lateral palmar nerve was dissected until its subdivision to originate the formation of the lateral and medial palmar metacarpal nerves, which were distally dissected, in their turn, until they emerged adjacent to the distal extremities of the metacarpal bones IV and II, respectively.

The anatomical nomenclature adopted was in accordance with the International Committee on Veterinary Gross Anatomical Nomenclature [13].

\section{RESULTS}

The lateral and medial palmar metacarpal nerves have been observed in all of the specimens, with an origin in the deep branch of the lateral palmar nerve, immediately distal to the intercarpal articulation. After its origin, the first nerve stretched ipsilaterally from the common trunk, whilst the second nerve positioned in a counter-lateral manner, after superficially crossing the interosseous muscle. Both nerves penetrated at the level of the mid third, between the mentioned muscle 
and the metacarpal bone III and would each take, in parallel, a position that was medial to the metacarpal bone IV and lateral to the metacarpal II, being intimately associated to the palmar face of the metacarpal bone III (Figure 1).

Distally, the lateral and medial palmar metacarpal nerves have respectively emerged, from a palmar to lateral and medial position at the level of the distal extremity of the corresponding metacarpal bones (IV and II) and adjacent to the palmar recess of the metacarpophalangeal articulation, where they had finally ramified to partially innervate the associated articular capsule and the subcutaneous tissue of the region (Figures $2 \mathrm{~A}$ and $3 \mathrm{~A}$ ).

As a part of the four-point diagnostic anesthetic block of the metacarpophalangeal articulation, for the desensitization of the lateral and medial palmar metacarpal nerves, the palpation of the distal extremities of the metacarpal bones IV and II is suggested. After the identification, whilst resorting to the thumb lodged from the extremity in a proximal direction, the needle is introduced $1.5 \mathrm{~cm}$ bilaterally, in a perpendicular position, between the aforementioned metacarpal bones and the interosseous muscle - the latter and the tendons of the flexor muscles being pulled in the palmar direction (Figures 2B \& 3B).

\section{DISCUSSION}

The anatomy of the distal third of the thoracic limb in equines is well known and is being extensively studied [20] with aims at a better comprehension of the biomechanics of locomotion, of how each ailment interacts with specific structures, and at the devising of new modes of diagnosis [17], with grounds on the high incidence of locomotor disorders in that region [19].

Out of a number of principles which support the use of the techniques of local anesthesia for diagnosis in that species, we may quote the good morpho-

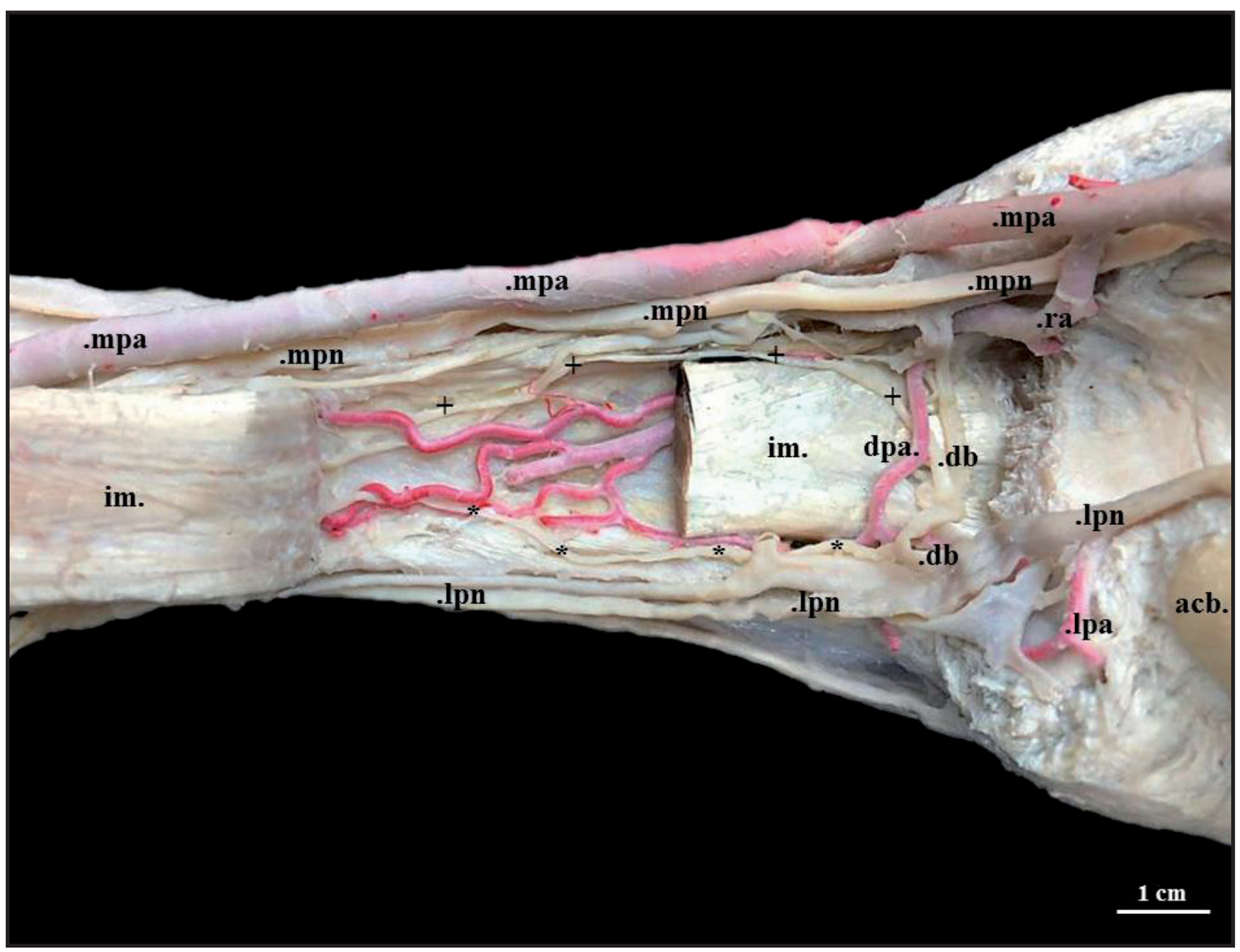

Figure 1. Palmar view of the proximal region of the right hand of an indeterminate equine breed, whilst highlighting the origin of the medial palmar metacarpal (+) and the lateral palmar metacarpal nerves $(*)$, as from the deep branch of the lateral palmar nerve (db). Other structures referenced: accessory carpal bone (acb); lateral palmar artery (lpa); lateral palmar nerve (lpn); radial artery (ra); medial palmar artery (mpa); medial palmar nerve (mpn), interosseous muscle (im); deep palmar arch (dpa). 
T.M. Vieira, H.I.R. Magalhães, Y.H. Paula, et al. 2019. Sites of Anesthetic Block of the Lateral and Medial Palmar Metacarpal Nerves in Equines of Indeterminate Breed.

Acta Scientiae Veterinariae. 47: 1661.
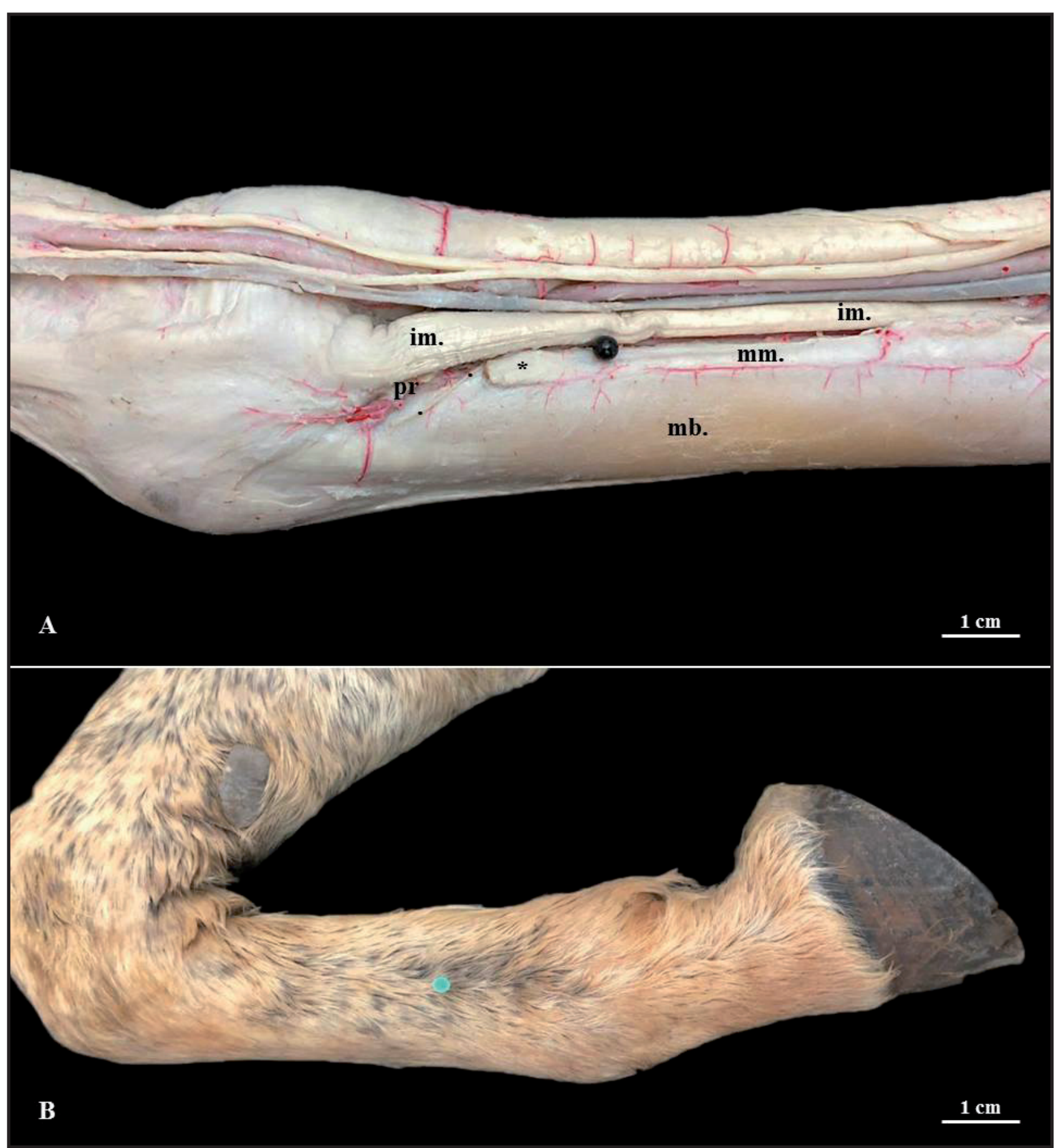

Figure 2. A- Medial view of the left hand of a indeterminate equine breed. The palmar-to-medial trajectory of the medial metacarpal palmar nerve is highlighted (.). The said nerve emerges in a region that is delimited by the following structures: metacarpal bone III (mb); metacarpal bone II (mm), and distal extremity of the metacarpal bone II $(*)$; interosseous muscle (im), and palmar recess of the metacarpophalangeal articulation (pr). Black pin representing the site of needle penetration. B- Medial view of the right hand of a indeterminate equine breed. Demonstration of introduction of the needle $(1.5 \mathrm{~cm})$ in a perpendicular and proximal orientation, between the metacarpal bone II and the interosseous muscle, after the identification of the distal extremity of the metacarpal bone II and the lateral-palmar pulling of the tendons of the flexor muscles. 
T.M. Vieira, H.I.R. Magalhães, Y.H. Paula, et al. 2019. Sites of Anesthetic Block of the Lateral and Medial Palmar Metacarpal Nerves in Equines of Indeterminate Breed.

Acta Scientiae Veterinariae. 47: 1661.
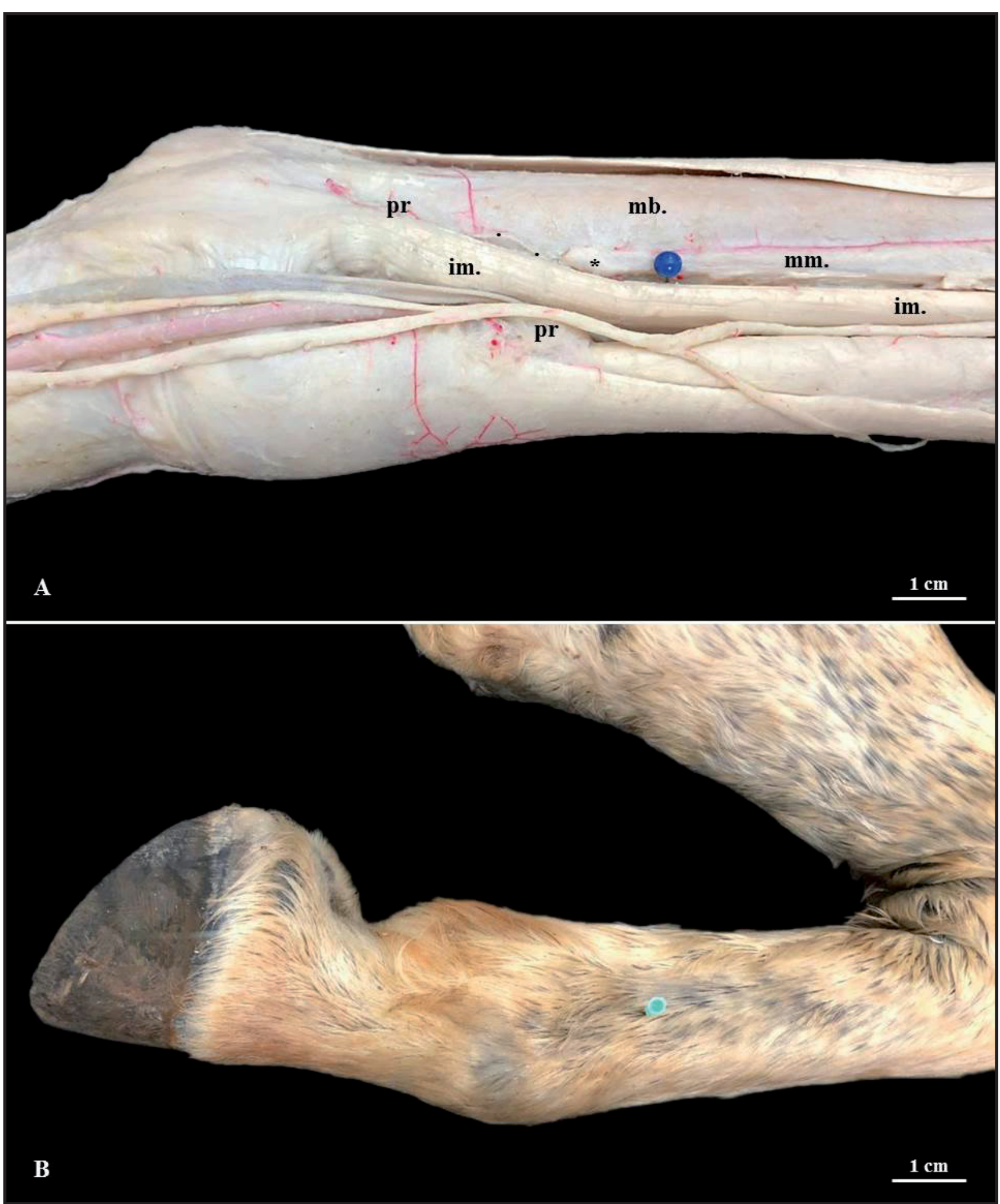

Figure 3. A- Lateral view of the left hand of a indeterminate equine breed. The palmar-to-lateral trajectory of the lateral metacarpal palmar nerve is highlighted (.). The said nerve emerges in a region that is delimited by the following structures: metacarpal bone III (mb); metacarpal bone IV (mm) and distal extremity of the metacarpal bone IV (*); interosseous muscle (im) and palmar recess of the metacarpophalangeal articulation (pr). Blue pin representing the site of needle penetration. B- Lateral view of the right hand of a indeterminate equine breed. Demonstration of introduction of the needle $(1.5 \mathrm{~cm})$ in a perpendicular and proximal orientation, between the metacarpal bone IV and the interosseous muscle, after identification of the distal extremity of the metacarpal bone IV and the medial-palmar pulling of the tendons of the flexor muscles. 
logical knowledge of the regions addressed, hence, without the understanding of the area innervated by each peripheral nerve and the possibility of diffusion of the anesthetic to the adjacent structures, the interpretation of the results is rendered impossible, whilst providing room for misplaced diagnoses [1].

In regard of the lateral and medial palmar metacarpal nerves, according to Getty [10] and Budras et al. [4], they were the continuation of the fibers of the deep branch of the lateral palmar nerve after a ramification that is distal to the carpus, and have positioned parallel [1] and "axially" to the metacarpal bones IV and II $[4,7,10]$ at the palmar surface of the metacarpal bone III [10].

Similarly, in equines of indeterminate breed, the lateral and medial palmar metacarpal nerves have been observed with their origins in the deep branch of the lateral palmar nerve. The mentioned nerves have respectively positioned in an ipsilateral and counter-lateral manner from their origins, and have subsequently penetrated at the level of the mid third, between the interosseous muscle and the metacarpal bone III. Nevertheless, the distribution observed for both nerves from that point on contradicts the description recorded in the aforementioned literature, hence they have been termed as "axial" in regard of two different bones (one lateral, and the other one medial).

Upon consultation to the Nomina Anatomica Veterinaria (N.A.V.) in force [13], it has been noticed that the terms axial and abaxial ought to be used as indicators of position and direction, only as a reference from the metacarpophalangeal and metatarsophalangeal articulations of those species whose functional axis of the limb passes between the digits III and IV, as in the case of artiodactyl unguligrade animals. Ergo, as has also been pointed out by Getty [10], we reiterate the inconvenience of using these terms for equines, as they are still widely applied in the scientific production and in the clinical routine.

Still within the context of indicative terms, the didactic heritage consecrated in animal anatomy $[7,10,15]$, including the very N.A.V. [13], have highlighted that a structure/surface that is closer to the median sagittal plane than another is referred to as medial, whilst the one that is closer to the plane of lateral delimitation is referred to as lateral. Then, we may affirm that, after associating to the palmar face of the metacarpal bone III, the lateral and medial palmar metacarpal nerves have, in parallel, taken a position that is medial to the metacarpal bone IV and lateral to the metacarpal bone II, respectively.

Yet, in the distal extremity of the corresponding metacarpal bones, the nerves studied have emerged from a palmar to lateral and medial position and, so, as stated by Dyce et al. [7] and Baccarin et al. [1], have become superficial to innervate the palmar and dorsal recesses of the metacarpophalangeal articulation [7] and the articular capsule in part [10].

The anesthetic blockage of these nerves, at the level of the distal extremity of the metacarpal bones IV and II, is carried out by introducing the needle parallel to the skin [1] and, together with the desensitization of the lateral and medial palmar nerves in this region [11], comprise the four-point anesthetic block of the metacarpophalangeal articulation [19], which will provide, if properly performed, the analgesia of the proximal region of this articulation and of the entire area that is distal to that point [11].

As advised in the literature, and visualized in this study as well, there is a proximity with which the lateral and medial palmar metacarpal nerves appear in the palmar recess of the metacarpophalangeal articulation [1], and the risk of complications involving these structures is remarkable when the neural anesthetic technique is performed as from the introduction of needles at the level of the distal extremity of the metacarpal bones IV and II. Therefore, a blockage performed in a moderately proximal manner, in relation to those which have already been described, could reduce the chances of lesions in result of this technique, and would not cause too much alteration in regard of the anesthetized region, so as to interfere with the sequence of the tests for the diagnosis of claudication.

This way, palpation is suggested for the distal extremities of the metacarpal bones IV and II and, afterwards, with use of the thumb lodged as from the extremity of the same, in a proximal direction, the needle is to be penetrated $1.5 \mathrm{~cm}$ bilaterally and perpendicularly between the aforementioned metacarpal bones and the interosseous muscle, with the latter and the tendons of the flexor muscles being pulled in the palmar direction, thus avoiding trauma to the structures nearby.

Thus, it becomes valid to affirm that the technique of four-point diagnostic anesthetic block of the metacarpophalangeal articulation has been perfected for equines, and that it will limit the chances of false 
results on account of misplaced puncturing of nearby structures, such as, for example, the synovial recesses that are mentioned by Baccarin et al. [1] as the location of the utmost gravity, hence septic synovitis may come in play.

\section{CONCLUSIONS}

The lateral and medial palmar metacarpal nerves did present origins and distribution similar to that which has already been described, however, though extensively mentioned in the literature, we highlight herein, as recommended by the Nomina Anatomica Veterinaria, the non-utilization of the terms axial and abaxial as indicators of position and direction for equines.

As a part of the four-point diagnostic anesthetic block of the metacarpophalangeal articulation for the desensitization of the nerves studied, the palpation of the distal extremities of the metacarpal bones IV and
II is suggest. After the identification, whilst resorting to the thumb lodged from the extremity in a proximal direction, the needle is introduced $1.5 \mathrm{~cm}$ bilaterally, in a perpendicular position, between the aforementioned metacarpal bones and the interosseous muscle - the latter and the tendons of the flexor muscles being pulled in the palmar direction, thus avoiding trauma to the structures nearby and perfecting the technique used.

\section{MANUFACTURERS}

${ }^{1}$ Artecola ${ }^{\circledR}$ Química. Campo Bom, RS, Brazil.

${ }^{2}$ Suvinil ${ }^{\circledR}$ Tintas e Pigmentos. São Bernardo do Campo, SP, Brazil.

${ }^{3} \mathrm{Chemco}^{\circledR}$ Indústria e Comércio. Hortolândia, SP, Brazil.

Ethical approval. The study had been approved by the Board of Ethics in Animal Use of the University Center of Patos de Minas (UNIPAM), protocol $n^{\circ} 18 / 17$.

Declaration of interest. The authors report no conflicts of interest. The authors alone are responsible for the content and writing of paper.

\section{REFERENCES}

1 Baccarin R.Y.A., Brossi P.M. \& Silva L.C.L.C. 2015. Guia ilustrado para injeção perineural em membros locomotores de equinos. São Paulo: Quiron Comunicação, 53p.

2 Baxter G.M. 2011. Adams \& Stashak's lameness in horses. 6th edn. Hoboken: Wiley-Blackwell, 1272p.

3 Birch H.L. \& Goodship A.E. 1999. Can appropriate training regimes reduce the incidence of skeletal injury and loss of horses from training? Equine Veterinary Education. 11(6): 310-313.

4 Budras K.D., Sack W.O. \& Röck S. 2009. Anatomy of the Horse. 5th edn. Hannover: Schlütersche, 208p.

5 Carrijo Junior O.A. \& Murad J.C.B. 2016. Animais de grande porte II. Brasília: Nt Editora, 192p.

6 Costa M.H.C.G. 2012. Incidência de Lesões Locomotoras no Cavalo, Diagnosticadas por Raio-X. 84f. Lisboa, Portugal. Dissertação (Mestrado em Medicina Veterinária) - Curso de Mestrado Integrado em Medicina Veterinária, Universidade Lusófona de Humanidades e Tecnologias.

7 Dyce K.M., Sack W.O. \& Wensing C.J.G. 2010. Textbook of Veterinary Anatomy. 4th edn. Saint Louis: Elsevier, $834 p$.

8 Dyson S. 1984. Nerve blocks and lameness diagnosis in the horse. In Practice. 6(4): 102-107.

9 Feitosa F.L.F. 2014. Semiologia Veterinária: A Arte do Diagnóstico. 3.ed. São Paulo: Roca, 627p.

10 Getty R. 1986. Sisson/Grossman Anatomia dos Animais Domésticos. 5.ed. Rio de Janeiro: Guanabara Koogan, 2000p.

11 Gibson K.T. \& Stashak T.S. 1989. Using perineural anesthesia to localize equine lameness. Veterinary Medicine. 84(11): 1082-1086.

12 Hinchcliff K.W., Kaneps A.J. \& Geor R.J. 2004. Equine Sports Medicine and Surgery. Philadelphia: Saunders Co., $1364 \mathrm{p}$.

13 International Committee on Veterinary Gross Anatomical Nomenclature. 2017. Nomina Anatomica Veterinaria. 6th edn. Hannover: Editorial Committee, 178p.

14 Keg P.R., van den Belt A.J.M., Merkens H.W., Barneveld A. \& Dik K.J. 1992. The effect of regional nerve blocks on the lameness caused by collagenase induced tendinitis in the medimetacarpal region of the horse: a study using gait analysis and ultrassonography to determine tendon healing. Transboundary and Emerging Diseases. 39(5): 349-364.

15 König H.E. \& Liebich H.G. 2016. Anatomia dos Animais Domésticos: Texto e Atlas Colorido. 6.ed. Porto Alegre: Artmed, 804p.

16 Moyer W., Schumacher J. \& Schumacher J. 2007. A Guide to Equine Joint Injection and Regional Anesthesia. Yardley: Veterinary Learning Systems, 111p. 
T.M. Vieira, H.I.R. Magalhães, Y.H. Paula, et al. 2019. Sites of Anesthetic Block of the Lateral and Medial Palmar Metacarpal Nerves in Equines of Indeterminate Breed. Acta Scientiae Veterinariae. 47: 1661.

17 Ribeiro G.H.C. 2013. Anatomia, Biomecânica e Principais Patologias do Membro Distal de Equinos: Quartela e Casco. 48f. Goiânia, GO. Seminários Aplicados - Programa de Pós-graduação em Ciência Animal, Escola de Veterinária e Zootecnia da Universidade Federal de Goiás.

18 Ross M.W. \& Dyson S.J. 2003. Diagnosis and Management of Lameness in the Horse. Saint Louis: Saunders Co., $1095 p$.

19 Stashak T.S. 2006. Claudicação em Equinos segundo Adams. 5.ed. São Paulo: Roca, 1112p.

20 Whitcomb M.B. 2009. Ultrasonographic Evaluation of the Distal Extremity. Journal of Equine Veterinary Science. 29(1): 47-59. 